\title{
Illumination in binaries ${ }^{\star}$
}

\author{
J.-M. Hameury ${ }^{1}$ and H. Ritter ${ }^{2}$ \\ 1 URA 1280 du CNRS, Observatoire de Strasbourg, 11 rue de l'Université, F-67000 Strasbourg, France \\ 2 Max-Plank-Institut für Astrophysik, Karl-Schwarzschild-Str. 1, 85740 Garching, Germany
}

Received July 10; accepted September 17, 1996

\begin{abstract}
We give a simple, but accurate method that can be used to account for illumination in compact binary systems which have a low-mass companion, even if spherically symmetric illumination of the secondary star (not necessarily on the main sequence) is not assumed. This is done by introducing a multiplicative factor $\Phi$ in the Stefan-Boltzmann surface boundary condition, which accounts for the blocking of the intrinsic secondary flux by X-ray heating of the photospheric layers. Numerical fits and tables for $\Phi$ are given for unperturbed effective temperatures in the range $2500-5600 \mathrm{~K}$ and $\log g$ in the range $1.0-5.0$.
\end{abstract}

Key words: accretion, accretion discs - stars: binaries: close - X-rays: stars - methods: numerical

\section{Introduction}

The effects of irradiation of the low mass companion in a low-mass X-ray binary or a cataclysmic variable have received much attention in the recent years. In cataclysmic variables, the external illumination flux due to accretion onto the primary compact object is comparable to the intrinsic stellar flux produced by nuclear reactions, whereas this external flux exceeds by orders of magnitude that of the secondary in low-mass X-ray binaries. A fraction of the illumination flux is absorbed in optically thin regions of the secondary photosphere, and this may result in the formation of a wind (see e.g. London et al. 1981; Ruderman et al. 1989; Tavani \& London 1993), which can in turn affect the long term evolution of the system. We shall not discuss this effect here, but instead consider energy deposition below the photosphere of the secondary.

Early calculations in the case of low-mass X-ray binaries (Podsiadlowski 1991; Harpaz \& Rappaport 1991) in which it was assumed that the secondary is illuminated in

Send offprint requests to: J.-M. Hameury

* Tables 1 and 2 are only available in electronic form at the CDS via anonymous ftp 130.79.128.5 a spherically symmetric manner showed a quite dramatic effect. The intrinsic luminosity of the secondary star is very efficiently blocked by the impinging radiation in the outer convective layers, so that the secondary must expand until it becomes almost fully radiative. The secular evolution is then quite different from the standard case, namely the systems rapidly evolve towards longer periods, with mass transfer rates close to or above the Eddington limit. This would have accounted for the fact that the period distribution of LMXBs seems to be significantly different from that of cataclysmic variables, showing a lack of systems at short periods. This would have also increased by a large factor the number of LMXBs, making it compatible with what is required to account for the observed density of millisecond pulsars which are believed to be the descendants of LMXBs (Frank et al. 1992). However, the spherically symmetric assumption is obviously incorrect, and it was later shown by Hameury et al. (1993) and confirmed by Harpaz \& Rappaport (1995) that the unilluminated side can efficiently cool the secondary. The secular evolution of LMXBs is not drastically different from the unilluminated case, although the short term behaviour is very significantly affected by illumination, and exhibits on and off states, with relatively high mass transfer rates.

In the simpler context of cataclysmic variables, Ritter et al. $(1995,1996 a)$ showed that irradiation-induced mass transfer cycles could also be present in these systems; King (1995) and King et al. $(1995,1996)$ discussed from a very general point of view the occurrence of such mass transfer cycles in CVs. The existence of these cycles could be responsible for the observed spreading of mass transfer rates $\dot{M}$ for a given orbital period $P$, whereas models would predict a fair correlation between $\dot{M}$ and $P$. Ritter et al. (1996b) showed that, in the bi-polytrope approximation (Kolb \& Ritter 1992), the stability criterion depends critically upon the variation of the effective temperature of the illuminated star as a function of the irradiating flux. For modeling the response of the stellar surface to illumination, they used a very simple one zone model for the superadiabatic layers of the low-mass secondary. Here, we use detailed stellar models to calculate the response of the 
secondary, namely the secondary luminosity as a function of irradiation, for a range of values of the surface gravity and unperturbed effective temperature. We give our results in a tabular form that can be used to calculate the evolution of a compact system in the presence of illumination. The advantage of this formulation is that it can be used in bi-polytropic codes, which are much faster that full stellar codes, and thus allow the exploration of a wide range of parameters, but have to be calibrated.

\section{Reaction of the stellar surface to external irradiation}

The influence of heating of subphotospheric layers by an external irradiation flux is entirely described by a modification of the boundary condition, as all of the absorbed X-ray flux is thermalized before being re-emitted. In the plane parallel approximation, the standard StefanBoltzmann law $L=4 \pi R_{2}^{2} \sigma T_{\text {eff }, 0}^{4}$ where $L$ is the surface luminosity of the secondary star in the absence of illumination (note that $L$ need not be equal to the nuclear luminosity), $R_{2}$ its radius, and $T_{\text {eff }, 0}$ is the effective temperature of the unilluminated star has to be replaced by (Ritter et al. 1996b):

$L=R_{2}^{2} \sigma T_{\text {eff }, 0}^{4} \int_{0}^{2 \pi} \int_{0}^{\pi} G(x(\theta, \phi)) \sin \theta \mathrm{d} \theta \mathrm{d} \phi$,

where $x=F_{\mathrm{irr}} / \sigma T_{\mathrm{eff}, 0}^{4}$ is the normalized illuminating flux, and

$G(x)=\left(\frac{T_{\text {eff }}(x)}{T_{\text {eff }, 0}}\right)^{4}-x$.

Here $T_{\text {eff }}$ is the effective temperature of a stellar surface element subject to the illuminating flux $x$. The function $G(x)$, in the range $0-1$, describes the blocking of the intrinsic stellar luminosity as a result of illumination. $F_{\text {irr }}$ includes only the fraction of the flux that is deposited below the photosphere; as mentioned above, energy deposition in optically thin regions does not result in modifications of the internal structure of the star.

In the case of low-mass secondaries which have a convective envelope, the entropy deep in the envelope remains constant over the whole surface of the star, and varies slowly with time, on the Kelvin-Helmholtz time of the whole envelope. By contrast, the superadiabatic and radiative outer layers have a very short thermal time, and are in thermal equilibrium. Moreover, Gontikakis \& Hameury (1993) have shown that the flux, in the plane parallel approximation, does not vary with depth in the convective zone. The problem of determining the reaction of the star to illumination thus reduces to finding the structure of an initially convective layer in thermal equilibrium when one changes the outer boundary condition while keeping the entropy at its base constant. This is easily done solving the

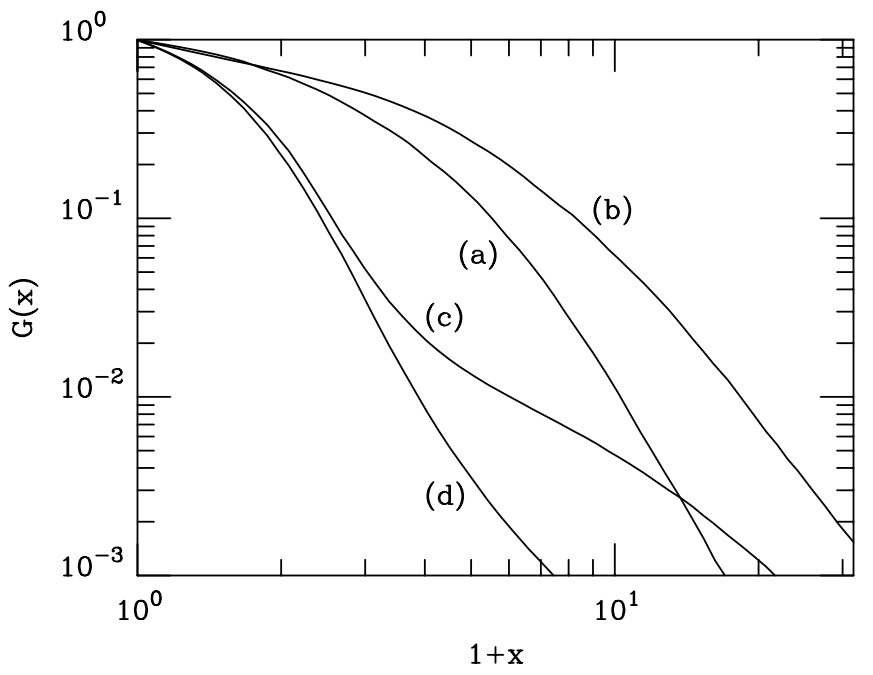

Fig. 1. Function $G(x)$ for secondaries on the main sequence, with a mass $0.8 \mathbf{a}), 0.6 \mathbf{b}), 0.4 \mathbf{c}$ ) and $0.2 M_{\odot} \mathbf{d}$ )

standard stellar structure equations, which, in the plane parallel approximation write:

$$
\begin{aligned}
& \frac{\mathrm{d} \log P}{\mathrm{~d} \log \Sigma}=g \\
& \frac{\mathrm{d} \log T}{\mathrm{~d} \log P}=\nabla,
\end{aligned}
$$

where $P$ is the pressure, $T$ the temperature, $\nabla$ the temperature gradient calculated using the mixing length approximation in the convective zone, $\Sigma$ the column density, and $g$ the surface gravity, assumed to be constant. The energy flux $F$ is also assumed to be constant throughout the layer. The layer is integrated down to a depth $\Sigma_{\max }$ of $10^{6} \mathrm{~g} \mathrm{~cm}^{-2}$, which is deep enough that the departure from adiabaticity is negligible; our results have been found to be independent of the particular value of $\Sigma_{\max }$. The surface boundary conditions are standard:

$\kappa P=2 / 3 g, \quad F+F_{\text {irr }}=\sigma T^{4}$.

In the absence of illumination, these equations are integrated for a given $g$ and $T_{\text {eff }}$, and give the entropy $S_{0}$ deep in the convective zone. In the presence of an irradiating flux, one adds the condition $S=S_{0}$ at the base of this layer. The set of Eq. (3) are integrated using the method, equation of state and opacities described in Hameury (1991). This gives $F$, and thus $G$ as a function of $g, T_{\text {eff, } 0}$, and $x$. Figure 1 shows $G(x)$ for main sequence stars of various masses. A significant difference can be seen between very low mass secondaries (less than $\left.0.4 M_{\odot}\right)$ and more massive ones. This difference is due to the low value of the energy flux that has to be carried through the convective zone, and hence to a small deviation to adiabaticity, even in the outer superadiabatic layers. For those low-mass stars, $G(x)$ is not very different 


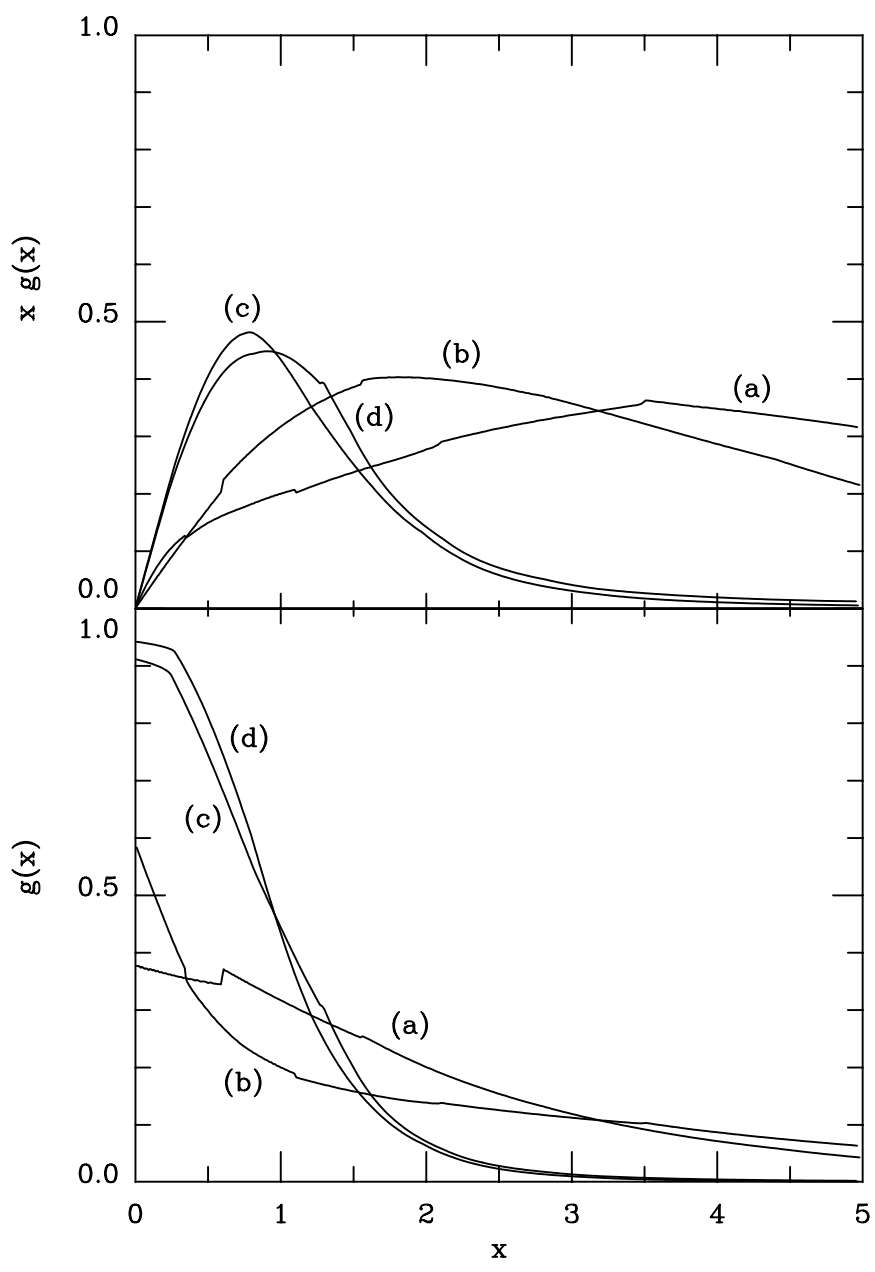

Fig. 2. Functions $g(x)=-\mathrm{d} G(x) / \mathrm{d} x$ and $x g(x)$ for secondaries on the main sequence with masses $0.8 \mathbf{a}), 0.6 \mathbf{b}), 0.4 \mathbf{c}$ ) and $0.2 M_{\odot} \mathbf{d}$ )

from the relation $G(x)=\max (1-x, 0)$ that one would obtain assuming that there is no superadiabatic region, so that the temperature gradient and thus the surface temperature is fixed.

The stability analysis of mass transfer involves the derivative of the function $G(x)$ (Ritter et al. 1996c), $g(x)=-\mathrm{d} G(x) / \mathrm{d} x$. More precisely, the criterion for stability against irradiation-induced mass transfer is:

$C=2\left(\zeta_{\mathrm{s}}-\zeta_{\mathrm{R}}\right) \frac{\tau_{\mathrm{KH}, 0}}{\tau_{\dot{M}}} \mathcal{F}^{-1}>2 \operatorname{sxg}(x)$

where $\zeta_{\mathrm{s}}$ is the adiabatic mass radius exponent of the secondary, $\tau_{\mathrm{KH}, 0}$ the Kelvin-Helmholtz time, $\tau_{\dot{M}}$ the mass transfer time scale, $\mathcal{F}$ a dimensioneless function which scales roughly as the inverse of the mass of the secondary convective envelope, $s \sim 1 / 2$ the fraction of the secondary exposed to illumination, and $\zeta_{\mathrm{R}}$ is the mass radius exponent of the Roche radius. This criterion is deduced from the assumption that the mass-radius exponent of the secondary under the sole influence of illumination must be less than $\zeta_{\mathrm{s}}-\zeta_{\mathrm{R}}$; Eq. (5) means that, for instability to occur, one must satisfy both conditions of sufficient illuminating fluxes and sensitivity of the secondary response to irradiation, i.e. large value of $g(x)$. $C$ does not depend on the very detailed structure of the illuminated star, and is easily accessible in the bi-polytropic approximation. The response of the secondary is entirely contained in the function $x g(x)$, which is plotted in Fig. 2. The small discontinuities are due to linear interpolations in determining the opacity. A remarkable characteristic of this function is that (1) $g(x)$ is a monotonously decreasing function, which is less than unity, and (2) that $x g(x)$ has a maximum of the order of 0.5 , whatever the secondary mass, although the position of this maximum does depend on it. Thus, there will be no cycles if $C>s$ (note that this is a sufficient but not necessary condition for stability).

It must be stressed that this procedure is valid only for stars which have a convective envelope, even though the whole star can be far out of thermal equilibrium. This would in fact be the main limitation in following the evolution of systems in which the effect of irradiation is so strong that at some point the star becomes fully radiative. This was a natural outcome of models assuming spherically symmetric illumination, but Hameury et al. (1991) have shown that this is no longer the case when one accounts for the asymmetry of irradiation.

These calculations can easily be generalized to stars which are not on the main sequence, provided they still have a convective envelope. Given the effective temperature $T_{\text {eff }}$ and surface gravity $g$, the set of Eq. (3) can be integrated in the absence of illumination and provide the unperturbed outer structure; the same procedure as previously is then applied. Table 1 (available only in electronic form at the CDS via anonymous ftp 130.79.128.5) gives $-\log _{10}(G)\left(\right.$ Col. 4) as a function of $\log g$ (Col. 1), $\log T_{\text {eff }}$ (Col. 2) and $\log (1+x)$ (Col. 3).

If one assumes that the irradiation source is a point located at the distance $a$ from the center of the secondary, Eq. (1) can be easily integrated over the whole stellar surface, which gives, following the notations of Ritter et al. (1996b):

$L=2 \pi R_{2}^{2} \sigma T_{\mathrm{eff}, 0}^{4}\left[1+f_{2}(q)+\int_{0}^{\theta_{\max }} G(x(\theta)) \sin \theta \mathrm{d} \theta\right]$

where $\theta$ is the colatitude with respect to the substellar point of a point on the secondary surface, $f_{2}(q)=R_{2} / a$, with $q=M_{2} / M_{1}$ the mass ratio, and the normalized irradiation flux $x$ is now given by:

$x(\theta)=x_{0} h(\theta)$

with

$x_{0}=\frac{\eta}{4 \pi} \frac{G M_{1} \dot{M}}{R_{1} a^{2}} / \sigma T_{\mathrm{eff}, 0}^{4}$ 


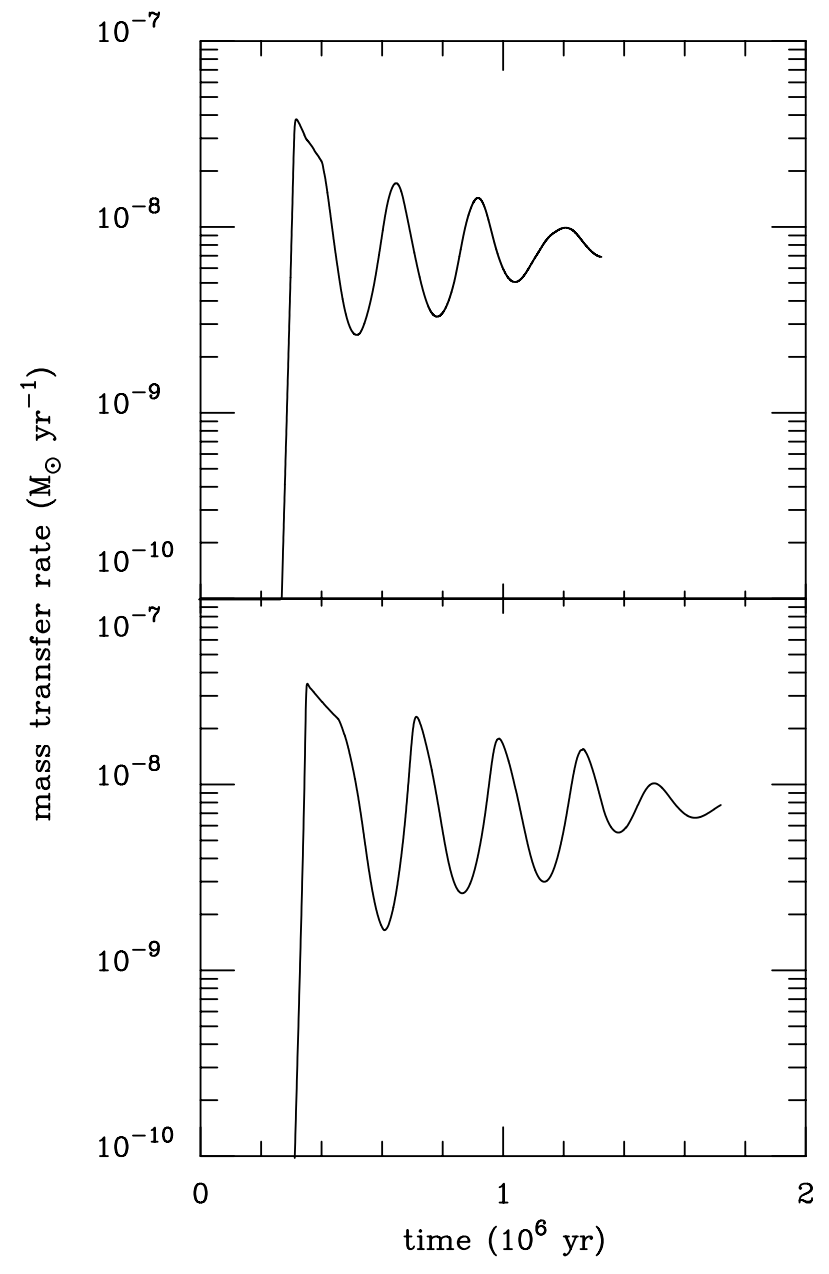

Fig. 3. Evolution of a cataclysmic variable using either a two hemispheres model with standard boundary conditions (top panel), or a spherically symmetric model with condition (9)

where $\eta$ is an efficiency factor accounting for the albedo of the secondary, the fraction of energy deposited in optically thin regions or the anisotropy of emission at the surface of the primary, and

$h(\theta)=\frac{\cos \theta-f_{2}}{\left(1-2 f_{2} \cos \theta+f_{2}^{2}\right)^{3 / 2}}$.

Equation (6) can thus be written in the form

$L=4 \pi R_{2}^{2} \sigma T_{\mathrm{eff}, 0}^{4} \Phi\left(x_{0}\right)$.

We have integrated numerically Eq. (6), and the results can be fitted within $1 \%$ by:

$$
\begin{aligned}
& y=\ln \left(1+x_{0}\right) \\
& \Phi\left(x_{0}\right)=\frac{1+f_{2}}{2}+\frac{1-f_{2}}{2} \exp \left(a_{1} y+a_{2} y^{2}+a_{3} y^{3}\right) .
\end{aligned}
$$

Table 2 (available only in electronic form at the CDS via anonymous ftp 130.79.128.5) gives the coefficients $a_{1}$, $a_{2}$, and $a_{3}$ as a function of $\log T_{\text {eff }, 0}$ and $\log g$. For arbitrary values of $\log T_{\text {eff }, 0}$ and $\log g$, a linear interpolation of $y$ (not of the coefficients $a_{1}, a_{2}$ and $a_{3}$ ) can be done.

The stability criterion now involves the integral of the function $x g(x)$; mass transfer will be stable if:

$C>\max \int_{0}^{\theta_{\max }} x(\theta) g(x(\theta)) \sin \theta \mathrm{d} \theta$.

We have calculated this maximum using the values of $G(x)$ as determined above, and found that this maximum is again almost independent of the secondary mass, and is smaller by about a factor 2 than the value $2 s$ found assuming uniform illumination over a fraction $s$ of the secondary, being equal to $(0.2-0.3)\left(1-f_{2}\right)$.

We have compared evolutionary calculations using either a spherically symmetric code with a boundary condition given by (10), or the code described in Hameury et al. (1993) in which both hemispheres with a coupling term are modeled. The results are given in Fig. 3, for a cataclysmic variable with $M_{1}=1 M_{\odot}$ and $M_{2}=0.8 M_{\odot}$; the white dwarf radius is $510^{8} \mathrm{~cm}$, and the efficiency $\eta$ is 0.1 (spherically symmetric model) and 0.09 (two hemispheres model). Both are very similar, i.e. the mass transfer rate exhibits damped oscillations; the different values of $\eta$ were chosen so as to obtain the same initial maximum value of the mass transfer rate. The differences (slightly shorter damping time and $\eta$ ) are mainly due to the fact that in the two hemispheres model, the illuminating flux is assumed to be constant over the heated regions, whereas the boundary condition (10) includes an angular variation of this flux, which has a strongly non-linear effect.

For X-ray luminosities close to the Eddington limit, we predict that the illumination effect is small, as $x g(x)$ is small for large values of $x$, and as a significant fraction of the secondary surface might be shielded from illumination by the accretion disc (Ritter et al. 1996b). However, Eq. (10) might significantly underestimate the effect of heating, as the heat flux in the illuminated regions can be negative, and be of the same order of magnitude as the intrinsic stellar flux. The unilluminated layers have then to re-radiate this additional flux, which may, in some cases, be sufficient to also block the intrinsic stellar flux. This is equivalent to having $x \sim 1$ in these regions which then become very sensitive to illumination. This effect, responsible for the short outbursts of mass transfer obtained by Hameury et al. (1993), is indeed not accounted for by the boundary condition (10), but depends sensitively on the strength of the coupling between both hemisphere, i.e. on the circulation timescale which is poorly known.

\section{Conclusion}

The effects of X-ray illumination of the secondary star in a CV or a LMXB can be accounted for by a modification of the Stefan-Boltzmann law which we give in a tabular form. This approximation is quite accurate in CVs, but may lead to a significant underestimate of the effects of 
illumination in LMXBs, when the luminosity is close to the Eddington limit. This modified boundary condition can be used in bi-polytropic codes, which are much faster than full stellar codes, and hence allow the exploration of a larger parameter space.

\section{References}

Frank J., King A.R., Lasota J.P., 1992, ApJ 385, L45

Gontikakis C., Hameury J.M., 1993, A\&A 271, 118

Hameury J.M., 1991, A\&A 243, 419

Hameury J.M., King A.R., Lasota J.P., Raison F., 1993, A\&A 277,81

Harpaz A., Rappaport S., 1991, ApJ 383, 739

Harpaz A., Rappaport S., 1995, A\&A 294, L49

King A.R., 1995, in: Bianchini A., Della Valle M. and Orio M. (eds.) Cataclysmic Variables. Kluwer, p. 523
King A.R., Frank K., Kolb U., Ritter H., 1995, ApJ 444, L37

King A.R., Frank K., Kolb U., Ritter H., 1996, ApJ 467, 761

Kolb U., Ritter H., 1992, A\&A 254, 213

London R., McCray R., Auer L.H., 1981, ApJ 243, 970

Podsiadlowski P., 1991 Nat 350, 136

Ritter H., Zhang Z., Kolb U., 1995, in: Bianchini A., della Valle M., Orio M. (eds.) Cataclysmic Variables. Kluwer, p. 479

Ritter H., Zhang Z., Kolb U., 1996a, in: Van Paradijs J., van den Heuvel E.P.J. and Kuulkers E. (eds.). IAU Symp. No. 165. Kluwer, p. 65

Ritter H., Zhang Z., Kolb U., 1996b, A\&A (to be submitted)

Ritter H., Zhang Z., Hameury J.M., 1996c, in: Evans A., Wood J.H. (eds.) Cataclysmic Variables and Related Objects, IAU Coll. No. 158. Kluwer, p. 449

Ruderman M., Shaham J., Tavani M., Eichler D., 1989, ApJ 343, 292

Tavani M., London R., 1993, ApJ 410, 281 